\title{
Coincidence Problem in YM Field Dark Energy Model
}

\author{
Wen Zhao* and Yang Zhang \\ Astrophysics Center, University of Science and Technology of China, Hefei, Anhui, China
}

\begin{abstract}
The coincidence problem is studied in the effective Yang-Mills condensate dark energy model. As the effective YM Lagrangian is completely determined by quantum field theory, there is no adjustable parameter in this model except the energy scale, and the cosmic evolution only depends on the initial conditions. For generic initial conditions with the YM condensate subdominant to the radiation and matter, the model always has a tracking solution, the Universe transits from matter-dominated into the dark energy dominated stage only recently $z \sim 0.3$, and evolve to the present state with $\Omega_{y} \sim 0.73$ and $\Omega_{m} \sim 0.27$.

PACS numbers: 98.80.-k, 98.80.Es, 04.30.-w, 04.62.+v
\end{abstract}

*wzhao7@mail.ustc.edu.cn 


\section{INTRODUCTION}

Recent observations on the Type Ia Supernova (SNIa) 1] , Cosmic Microwave Background Radiation (CMB) 2] and Large Scale Structure (LSS) 3] all suggest a flat Universe consisting of dark energy $(73 \%)$, dark matter $(23 \%)$ and baryon matter $(4 \%)$. How to understand the physics of the dark energy is an important issue, having the equation of state $\omega<-1 / 3$ and causing the recent accelerating expansion of the Universe. The simplest model is the cosmological constant $\Lambda$ with $\omega_{\Lambda}=-1$ (which can be viewed as the vacuum energy) and fits the observation fairly well. However, throughout the history of the Universe, the densities of matter and vacuum energy evolve differently, so it appears that the conditions in the early Universe have to be set very carefully in order for the energy densities of them to be comparable today. This is the coincidence problem [4]. Another one, called the "fine-tuning" problem, is why the present vacuum energy density is very tiny compared to typical scales in particle physics. These problems have stimulated a number of approaches to build the dark energy models with a dynamic field.

One class of approaches to the dark energy is to introduce dynamic scalar fields with a tracker behavior. The scalar fields are subdominant during early stages of expansion, and at late times, they run into the attractor behaving as the dark energy, and dominate over the matter component. The transition to the state $\omega \sim-1$ usually happens during the matter dominated era. This kind of models include quintessence [5] [6], k-essence [7], phantom [8], quintom[9] etc. The quintessence fields have attractor-like solutions converging to a common, cosmic evolutionary track for a very wide range of initial conditions rapidly. The initial value of $\rho_{\phi}$ can vary by many orders of magnitude without altering the cosmic history. The initial dark energy fraction after inflation can be as large as $\Omega_{\phi} \sim 10^{-3}$, which is natural in terms of the equipartition of energy among all the dynamic degrees of freedom[6]. The k-essence fields feature a tracker behavior during radiation dominated era, and a $\Lambda$-like behavior shortly after the transition to matter domination [7], but the initial dark energy fraction only occupies a very narrow region in the phase space [10].

The effective YM field condensate model has been introduced to describe the dark energy [11, 12, 13]. It has interesting features: the YM fields are the indispensable cornerstone to particle physics, gauge bosons have been observed. There is no room for adjusting the form of effective YM Lagrangian as it is predicted by quantum corrections according to field theory. In this paper, we examine the the coincidence problem in this model. We find that the YM condensate field was subdominant during earlier stages with a state of $\omega \sim 1 / 3$, and later it turned into a state of $\omega \sim-1$ and only recently it started to dominate over the matter. Moreover, the initial value of 
YM energy fraction can be taken in a wide range of $\Omega_{y} \simeq\left(10^{-20}, 10^{-2}\right)$. Thus the model can solve the problem naturally.

\section{THE EFFECTIVE YANG-MILLS FIELD MODEL}

The effective YM condensate cosmic model has been discussed in Ref. 11, 12, 13]. The effective Lagrangian up to 1-loop order is [14, 15]

$$
\mathrm{E}_{e f f}=\frac{b}{2} F \ln \left|\frac{F}{e \kappa^{2}}\right|,
$$

where $b=11 N / 24 \pi^{2}$ for the generic gauge group $S U(N)$ is the Callan-Symanzik coefficient [16] $F=-(1 / 2) F_{\mu \nu}^{a} F^{a \mu \nu}$ plays the role of the order parameter of the YM condensate, $e \simeq 2.72, \kappa$ is the renormalization scale with the dimension of squared mass, the only model parameter. The attractive features of this effective YM Lagrangian include the gauge invariance, the Lorentz invariance, the correct trace anomaly, and the asymptotic freedom[14]. With the logarithmic dependence on the field strength, $\mathrm{E}_{\text {eff }}$ has a form similar to he Coleman-Weinberg scalar effective potential[17], and the Parker-Raval effective gravity Lagrangian[18]. The effective YM condensate was firstly put into the expanding Robertson-Walker (R-W) spacetime to study inflationary expansion [11] and the dark energy [12]. We work in a spatially flat R-W spacetime with a metric

$$
d s^{2}=a^{2}(\tau)\left(d \tau^{2}-\delta_{i j} d x^{i} d x^{j}\right)
$$

where $\tau=\int\left(a_{0} / a\right) d t$ is the conformal time. Assume that the Universe is filled with the YM condensate. For simplicity we study the $S U(2)$ group and consider the electric case with $B^{2} \equiv 0$. The energy density and pressure are given by

$$
\rho_{y}=\frac{E^{2}}{2}(\epsilon+b), \quad p_{y}=\frac{E^{2}}{2}\left(\frac{\epsilon}{3}-b\right)
$$

where the dielectric constant is given by

$$
\epsilon=b \ln \left|\frac{F}{\kappa^{2}}\right| .
$$

and the equation of state $(\mathrm{EoS})$ is

$$
\omega=\frac{p_{y}}{\rho_{y}}=\frac{\beta-3}{3 \beta+3}
$$

where $\beta \equiv \epsilon / b=\ln \left|\frac{E^{2}}{\kappa^{2}}\right|$. At the critical point with the condensate order parameter $F=\kappa^{2}$, one has $\beta=0$ and $\omega=-1$, the Universe is in exact de Sitter expansion [11]. Around this critical 
point, $F<\kappa^{2}$ gives $\beta<0$ and $\omega<-1$, and $F>\kappa^{2}$ gives $\beta>0$ and $\omega>-1$. So in the YM field model, EoS of $\omega>-1$ and $\omega<-1$ all can be naturally realized. When $\beta \gg 1$, the YM field has a state of $\omega=1 / 3$, becoming a radiation component. As is known, an effective theory is a simple representation for an interacting quantum system of many degrees of freedom at and around its respective low energies. Commonly, it applies only in low energies. However, it is interesting to note that the YM condensate model as an effective theory intrinsically incorporates the appropriate states for both high and low temperature. As has been shown above, the same expression in Eq.(3) simultaneously gives $p_{y} \rightarrow-\rho_{y}$ at low energies, and $p_{y} \rightarrow \rho_{y} / 3$ at high energies. Therefore, our model of effective YM condensate can be used even at higher energies than the renormalization scale $\kappa$.

The effective YM equations are

$$
\partial_{\mu}\left(a^{4} \epsilon F^{a \mu \nu}\right)+f^{a b c} A_{\mu}^{b}\left(a^{4} \epsilon F^{c \mu \nu}\right)=0,
$$

the $\nu=0$ component of which is an identity, and the $\nu=1,2,3$ spatial components of which reduce to

$$
\partial_{\tau}\left(a^{2} \epsilon E\right)=0
$$

At the critical point $(\epsilon=0)$, this equation is an identity. When $\epsilon \neq 0$, this equation has an exact solution as follows [13],

$$
\beta e^{\beta / 2} \propto a^{-2}
$$

where the coefficient of proportionality in the above depends on the initial condition. Near the critical point with $|\omega+1| \ll 1$, i.e. $\beta \ll 1$, Eq.(8) yields

$$
\beta \propto a^{-2}
$$

and the EoS is

$$
\omega+1 \simeq \frac{4 \beta}{3} \propto a^{-2}
$$

This simple analysis shows that with the expansion of the Universe $\omega$ will goes to the critical point of $\omega=-1$, an important character of the YM dark energy model. The YM condensate can achieve the states of $\omega>-1$ and $\omega<-1$, but it can not cross over -1 , just like in the scalar models [19]. At the early stages, $a \rightarrow 0$, Eq.(8) leads to $\beta \gg 1$, and Eq.(15) gives $\omega \rightarrow 1 / 3$, so the YM condensate behaves as the radiation component. 
We should fix the value of $\kappa$, the only parameter in our model. At the present time, the YM energy density

$$
\rho_{y}=\frac{b E^{2}}{2}(\beta+1) \simeq \frac{b \kappa^{2}}{2},
$$

and, as the dark energy, it should be $\Omega_{y} \rho_{0}$, where the present total energy density in the Universe $\rho_{0} \approx 8.099 h^{2} \times 10^{-11} \mathrm{eV}^{4}$. We choose $\Omega_{y}=0.73$ as has been observed, yielding

$$
\kappa=3.57 h \times 10^{-5} \mathrm{eV}^{2} .
$$

This energy scale is low compared to typical energy scales in particle physics. So the "fine-tuning" problem is also present in this model.

To be more specific about how the YM condensate evolves in the expanding Universe, we look at an early stage when the Big Bang nucleosynthesis (BBN) processes occur around a redshift $z \sim 10^{10}$ with an energy scale $\sim 1 \mathrm{MeV}$. To see how the evolution of $\rho_{y}$ depends the the initial condition, we introduce the ratio of energies of the two components

$$
\left.r_{b} \equiv \frac{\rho_{y}}{\rho_{r}}\right|_{z=10^{10}},
$$

where $\rho_{r}$ is the radiation energy density. We consider $r_{b}<1$, i.e. the YM condensate is subdominant to the radiation component initially. Of course, the YM condensate evolves differently for different initial values of $r_{b}$. Nevertheless, we will see that, as the result of evolution, the present Universe is always dominated by the YM condensate $\Omega_{y} \sim 0.73$ for a very wide range of initial values $r_{b}$.

Now we use the exact solution (8) to plot the evolution of $\rho_{y}$ as a function of the redshift $z$ in Fig.[1]. As specific examples, here we take $r_{b}=10^{-2}$, and $r_{b}=10^{-20}$. In comparison, also plotted are the energy densities of radiation, and of matter. It is seen that, in the early stages, $\rho_{y}$ decreases as $\rho_{y} \propto a^{-4}$. So the YM density is subdominant and tracks the radiation, a scaling solution. The corresponding EoS of YM field is $\omega \simeq 1 / 3$ shown in Fig.[2]. At late stages, with the expansion of the Universe, $a \rightarrow \infty, \beta$ decreases to nearly zero, and $\omega \rightarrow-1$ asymptotically. Moreover, this asymptotic region is arrived at some redshift $z$ before the present time, and this $z$ has different values for different initial values of $r_{b}$. For smaller $r_{b}$, the transition redshift is larger (seen in Fig.[2]), and the transition happens earlier. Once the asymptotic region is achieved, the density of the YM field levels off and remain a constant forever, like a cosmological constant. We have also checked that the present value $\Omega_{y} \sim 0.73$ is also the outcome of the cosmic evolution for any value of $r_{b}$ in the very wide range $\left(10^{-20}, 10^{-2}\right)$. So the coincidence problem do not exist in the YM condensate model. 


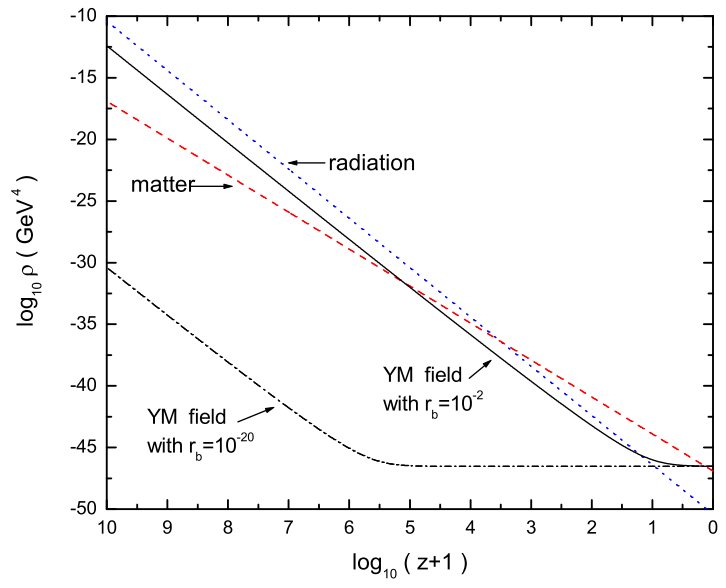

FIG. 1: The evolution of the energy densities versus redshift $z$. The dot line is for the radiation density, and the dash line for the matter density. The solid line is for the YM energy density with $r_{b}=10^{-2}$, and the dash-dot one for the YM energy density with $r_{b}=10^{-20}$.

The present value of $\omega_{0}$ is nearly -1. Fig. [3] plots the dependence of the present EoS $\omega_{0}$ on the initial condition $r_{b}$. The function $\log _{10}\left(r_{b}\right)$ versus $\log _{10}\left(\omega_{0}+1\right)$ is nearly linear: a smaller $r_{b}$ leads to a smaller $\omega_{0}$. For a value $r_{b}=10^{-2}$, one has $\omega_{0}=-0.99$. For a value $r_{b}=10^{-20}$, $\omega_{0}$ would be -1 accurately up to one in $10^{11}$. Therefore, at present the YM condensate is very similar to the cosmological constant.

The solution in Eq.(8) can converted into the following form

$$
z=\sqrt{\frac{\beta}{\beta_{0}}} \exp \left[\frac{\beta-\beta_{0}}{4}\right]-1,
$$

where $\beta_{0}$ is the value of $\beta$ at $z=0$, depending on the initial value $r_{b}$. For a fixed $\beta_{0}$, this formula tells a one-one relation between the $\operatorname{EoS}$ (through $\beta$ ) and the corresponding redshift $z$. As is seen from Fig.[2], the transition of $\omega$ from $1 / 3$ to -1 occurs during a finite period of time, instead of instantly. To characterize the time of transition, we use $z_{t}$ to denote the redshift when $\omega=-1 / 3$, i.e. $\beta=1$, as given by Eq.(5). This is, in fact, the time when the strong energy condition begins to be violated, i.e., $\rho_{y}+3 p_{y} \leq 0$. Then

$$
z_{t}=\sqrt{\frac{1}{\beta_{0}}} \exp \left[\frac{1-\beta_{0}}{4}\right]-1
$$

Therefore, this gives a function $z_{t}=z_{t}\left(r_{b}\right)$. Fig.[4] shows how the transition redshift $z_{t}$ depends on the ratio $r_{b}$. Interestingly, this transition can occur before, or after the radiation-matter equality 


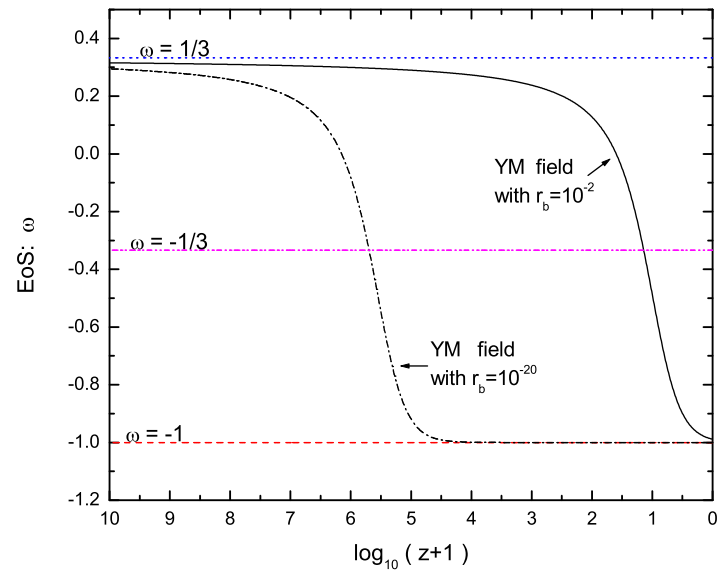

FIG. 2: The evolution of the equation of state $\omega$ for the YM field as a function of redshift $z$. Here the two curves are for the same models as those in Fig. 1.

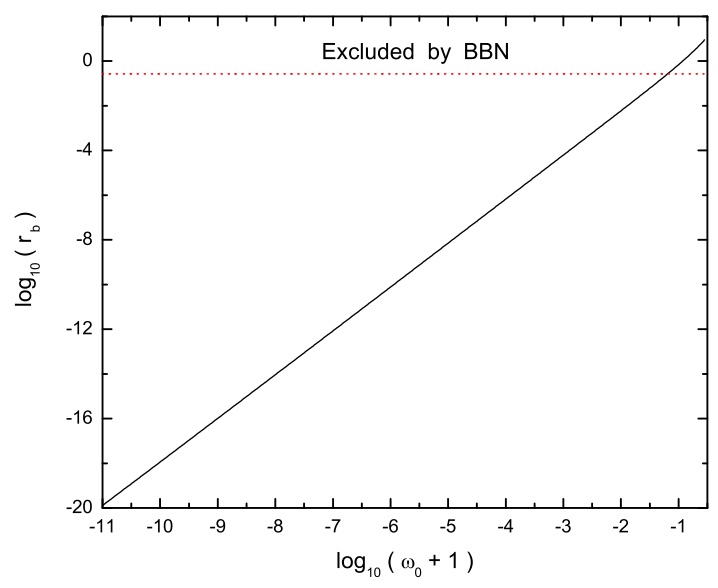

FIG. 3: The present EoS of the YM field $\omega_{0}$ depends on the initial value $r_{b}$. The region above the dot line has been excluded by the observation of the BBN [21].

$\left(z_{e q}=3233[2]\right)$. This feature is different from the tracked quintessence or k-essence models in which transition occurs during the matter dominated era [6] [7]. A larger $r_{b}$ leads to a smaller $z_{t}$. For example, $r_{b}=10^{-2}$ leads to $z_{t} \simeq 12.4 \ll z_{e q}$, and the transition occurs in the matter dominated stage, and $r_{b}=10^{-20}$ leads to $z_{t} \simeq 5.0 \times 10^{5} \gg z_{e q}$, and the transition occurs in the 


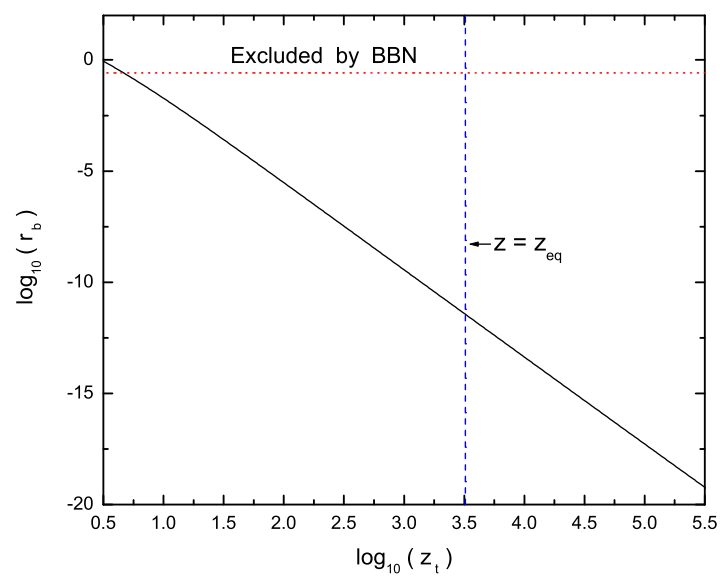

FIG. 4: The transition redshift $z_{t}$ depends on the initial value $r_{b}$. The region above the dot line has been excluded by the observation of the BBN [21].

radiation dominated stage.

The value of $r_{b}$ can not be chosen to arbitrarily large. In fact, there is a constraint from the observation result of the BBN. As is known, the presence of dark energy during nucleosynthesis epoch will speedup the expansion, enhancing the effective species $N_{\nu}$ of neutrinos [20, 21]. The latest analysis gives a constrain on the extra neutrino species $\delta N_{\nu} \equiv N_{\nu}-3<1.60$ [21]. Here in our model, the dark energy is played by the YM field. By a similar analysis, the ratio $r_{b}$ is related to $\delta N_{\nu}$ through $r_{b}=\frac{7 \delta N_{\nu} / 4}{10.75}$. This leads to an upper limit $r_{b}<0.26$, the present EoS $\omega_{0}<-0.94$ by Fig. [3], and the transition redshift $z_{t}>5.8$ by Fig. [4]. The range of initial conditions $r_{b} \in\left(10^{-20}, 10^{-2}\right)$ that we have taken satisfies this constraint.

\section{SUMMARY}

The effective YM condensate has the advantageous characters: the YM fields are indispensable to particle physics, there is no room for adjusting the functional form of the Lagrangian as it is predicted by quantum field theory. As a model for the cosmic dark energy, it has no free parameters except the present cosmic energy scale, and the cosmic evolution only depends on the initial conditions. This study has shown that, for a generic value $r_{b}$ in a wide range of $\left(10^{-20}, 10^{-2}\right)$, the present YM dark energy will be in $\left|\omega_{0}+1\right|<0.01$, and the Universe has $\Omega_{y} \sim 0.73$ and $\Omega_{m} \sim 0.27$ 
as the result of cosmic evolution. Thus, the YM model intrinsically has the tracking solution as in scalar field models. In this sense the model can naturally solve the coincidence problem.

It is found that at the early stages the subdominant YM field behaves like a relativistic radiation with $\omega \simeq 1 / 3$. Later around a redshift $z_{t}$, the YM field transits into the states $\omega \sim-1$, and only recently it becomes dominant and drives the current accelerating expansion of the Universe. Interestingly, the YM field model differs from the scalar models in that, the transition of the state from $\omega \sim 1 / 3$ to $\omega \sim-1$ can occur during radiation or matter dominated era, depending on the initial condition $r_{b}$. Finally, the observation of BBN gives a constraint $r_{b}<0.26$, which is not stringent. We have to say that the "fining-tuning" problem is not solved here as in most of other current models.

ACKNOWLEDGMENT: W.Zhao's work has been partially supported by Graduate Student Research Funding from USTC, and Y. Zhang's research work has been supported by the Chinese NSF (10173008), NKBRSF (G19990754), and by SRFDP.

[1] A.G.Riess et al., Astron.J. 116, 1009 (1998); S.Perlmutter et al., Astrophys.J. 517, 565 (1999); J.L.Tonry et al., Astrophys.J. 594, 1 (2003); R.A.Knop et al., Astrophys.J. 598, 102 (2003);

[2] C.L.Bennett et al., Astrophys.J.Suppl. 148, 1 (2003); D.N.Spergel et al., Astrophys.J.Suppl. 148, 175 (2003); D.N.Spergel et al., arXiv:astro-ph/0603449;

[3] M.Tegmark et al., Astrophys.J. 606, 702 (2004), Phys.Rev.D 69, 103501 (2004); A.C.Pope et al., Astrophys.J. 607, 655 (2004); W.J.Percival et al., MNRAS 327, 1297 (2001);

[4] P.Steinhardt, in 'Critical Problems in Physics' ed. by V.L.Fitch and D.R.Marlow (Princeton U.Press, 1997); R.H.Dicke and P.J.E.Peebles, in 'General Relativity: An Einstein Centenary Survey', ed. by S.W.Hawking \& W.Israel (Cambridge U. Press, 1979); E.J.Copeland, M.Sami and S.Tsujikawa, arXiv:hep-th/0603057,

[5] C.Wetterich, Nucl.Phys.B 302, 668 (1988); Astron.Astrophys. 301, 321 (1995); B.Ratra and P.J.E.Peebles, Phys.Rev.D 37, 3406 (1988); R.R.Caldwell, R.Dave and P.J.Steinhardt, Phys.Rev.Lett. 80, 1582 (1998);

[6] I.Zlatev, L.Wang and P.J.Steinhardt Phys.Rev.Lett. 82, 896 (1999); P.J.Steinhardt, L.Wang and I.Zlatev, Phys.Rev.D 59, 123504 (1999);

[7] C.Armendariz-Picon, T.Damour and V.Mukhanov, Phys.Lett.B 458, 209 (1999) ; T.Chiba, T.Okabe and M.Yamaguchi, Phys.Rev.D 62, 023511 (2000); C.Armendariz-Picon, V.Mukhanov and P.J.Steinhardt, Phys.Rev.D 63, 103510 (2001); T.Chiba, Phys.Rev.D 66, 063514 (2002);

[8] R.R.Caldwell, Phys.Lett.B 545, 23 (2002); S.M.Carroll, M.Hoffman and M.Trodden, Phys.Rev.D 
68, 023509 (2003); R.R.Caldwell, M.Kamionkowski and N.N.Weinberg, Phys.Rev.Lett. 91, 071301 (2003); M.P.Dabrowski, T.Stachowiak and M.Szydlowski, Phys.Rev.D 68, 103519 (2003); V.K.Onemli and R.P.Woodard, Phys.Rev.D 70, 107301 (2004);

[9] W.Hu, Phys.Rev.D 71, 047301 (2005); B.Feng, X.L.Wang and X.M.Zhang, Phys.Lett.B 607, 35 (2005); W. Zhao and Y. Zhang, Phys.Rev.D 73, 123509 (2006)

[10] M.Malquarti, E.J.Copeland and A.R.Liddle, Phys.Rev.D 68, 023512 (2003);

[11] Y.Zhang Phys.Lett.B 340, 18 (1994); Chin.Phys.Lett.14, 237 (1997);

[12] Y.Zhang, Gen. Rel. Grav. 34, 2155 (2002); Gen. Rel. Grav. 35, 689 (2003); Chin.Phys.Lett.20, 1899 (2003); Chin.Phys.Lett.21, 1183 (2004);

[13] W.Zhao and Y Zhang, Class.Quant.Grav. 23, 3405 (2006); arXiv:astro-ph/0508010

[14] H.Pagels and E.Tomboulis, Nucl.Phys.B 143, 485 (1978);

[15] S.Adler, Phys.Rev.D 23, 2905 (1981); Nucl.Phys.B 217, 3881 (1983);

[16] H.Politzer, Phys.Rev.Lett. 30, 1346 (1973); D.J.Gross and F.Wilzcek, Phys.Rev.Lett. 30, 1343 (1973);

[17] S.Coleman and E.Weinberg, Phys.Rev.D 7, 1888 (1973);

[18] L.Parker and A.Raval, Phys.Rev.D 60, 063512 (1999);

[19] A.Vikman, Phys.Rev.D 71, 023515 (2005);

[20] M.Birkel and S.Sarkar, Astropart.Phys. 6, 197 (1997); P.G.Ferreira and M.Joyce, Phys.Rev.D 58, 023503 (1998); J.P.Kneller and G.Steigman, Phys.Rev.D 67, 063501 (2003);

[21] R.H.Cyburt, B.D.Fields, K.A.Olive and E.Skillman, Astropart.Phys. 23, 313 (2005). 